\title{
Atividade antibacteriana do óleo essencial do manjericão frente a sorogrupos de Escherichia coli enteropatogênica isolados de alfaces
}

\author{
Antibacterial activity of essential basil oil against serogroups of enteropathogenic \\ Escherichia coli isolated from lettuce
}

\author{
André Gustavo Lima de Almeida Martins ${ }^{\mathrm{I}^{*}}$ Adenilde Ribeiro Nascimento $^{\mathrm{I}}$ \\ João Elias Mouchrek Filho' ${ }^{\mathrm{I}}$ Nestor Everton Mendes Filho' ${ }^{\mathrm{I}}$ Antonio Gouveia Souza \\ Natanael Eudes Aragão ${ }^{\mathrm{III}}$ Diôgo Sérgio Vieira da Silva ${ }^{\mathrm{I}}$
}

\section{RESUMO}

Considerando-se a crescente participação dos vegetais na transmissão de microrganismos, incluindo as Escherichia coli enteropatogênicas multirresistentes às drogas convencionais, a busca por novos compostos com propriedades antimicrobianas a partir de fontes naturais, tais como os óleos essenciais, tem se intensificado nos últimos anos. Esta pesquisa objetivou avaliar a atividade antibacteriana do óleo essencial do manjericão (Ocimum basilicum Linn.) frente a sorogrupos de Escherichia coli enteropatogennicas (EPEC) isolados de alfaces (Lactuca sativa), utilizando-se o Método de Difusão em Disco (MDD). Os resultados evidenciaram que todas as cepas de E. coli EPEC testadas apresentaram sensibilidade à ação do óleo essencial, sugerindo que este possa ser uma fonte de compostos com potencial terapêutico no combate a bactérias patogênicas.

Palavras-chave: Escherichia coli EPEC, óleo essencial, manjericão, atividade antibacteriana.

\section{ABSTRACT}

Considering the increase of the involvement of vegetables in the transmission of microorganism including the enteropathogenic Escherichia coli multiresistance to conventional drugs, the search for new antimicrobial compounds from natural sources, such as the essential oils, has been intensified in the last years. This research aimed to evaluate the antibacterial activity of the essential oil from basil (Ocimum basilicum Linn.) front to sorogroups of enteropathogenic Escherichia coli (EPEC) isolated in lettuces (Lactuca sativa) using the Diffusion in Disk Method. The results showed that, all the tested strains of $\mathbf{E}$. coli EPEC presented sensitivity to the essential oil's action, suggesting that it can be a source of compounds with therapeutic potential in the fighting to bacteria pathogenic.

Key words: Escherichia coli EPEC, essential oil, basil; antibacterial activity.

\section{INTRODUÇÃO}

O uso de plantas medicinais para o tratamento de doenças está associado à medicina popular de diferentes partes do mundo. Entre os principais produtos de origem vegetal com atividade antimicrobiana estão os extratos e os óleos essenciais (SARTORATTO et al., 2004). Entre as plantas medicinais comumente usadas na medicina popular está o manjericão (Ocimum basilicum Linn.), o qual pertence à família Lamiaceae, possuindo espécies originárias de regiões tropicais e subtropicais da Ásia, da África e da América Central e do Sul (LABRA et al., 2004).

A composição dos óleos essenciais extraídos das folhas e dos ápices com inflorescência do manjericão varia de acordo com a espécie e a localização geográfica, sendo classificados em quatro quimiotipos, de acordo com os componentes majoritários do óleo: quimiotipo linalol-metil chavicol (Europeu), metil chavicol (Reunião), metil cinamato

\footnotetext{
IDepartamento de Tecnologia Química, Universidade Federal Maranhão (UFMA), Campus Universitário do Bacanga, Av. dos Portugueses, S/N, 65085-580, São Luís, MA, Brasil. E-mail: andremartins1975@yahoo.com.br. *Autor para correspondência. "Laboratório de Combustíveis-LACOM, Universidade Federal da Paraíba (UFPB), João Pessoa, PB, Brasil.

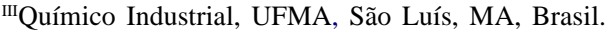


(Tropical) e quimiotipo eugenol (Java). Em função de suas propriedades terapêuticas, o manjericão é amplamente utilizado na medicina popular como antiespasmódico, antitérmico, auxiliando também na digestão e no combate de infecções bacterianas e parasitárias intestinais (TELCI et al., 2006).

A atividade antimicrobiana do óleo essencial do manjericão tem sido relatada como sendo predominantemente associada aos seus constituintes majoritários, o metil chavicol e o linalol. O óleo essencial dessa planta tem sido utilizado nas indústrias de alimentos como flavorizante para produtos de confeitaria, bebidas não alcoólicas, sorvetes e como condimentos e na indústria de cosméticos (BOZIN et al., 2006).

O hábito alimentar da população tem mudado consideravelmente, e o aumento no consumo de hortaliças in natura caracteriza essa mudança. Nesse contexto, a alface (Lactuca sativa) está entre as hortaliças folhosas mais consumidas no Brasil, sendo um dos componentes mais presentes nas saladas dos brasileiros. Seu cultivo é praticado de forma orgânica, livre de pesticidas ou agrotóxicos; hidropônica, em que as hortaliças ficam protegidas contra diversos fatores adversos do meio ambiente, como chuvas, geadas e ventos fortes, favorecendo a produtividade do produto; e convencional, com utilização de defensivos agrícolas (SANTANA et al., 2006).

Segundo FERNANDES et al. (2002), as hortaliças, por serem consumidas in natura, têm sido consideradas como uma das principais veiculadores de microrganismos patogênicos de interesse em Saúde Pública. Esses autores relatam ainda que as principais fontes de contaminação de hortaliças são a água de irrigação, que pode apresentar uma grande quantidade de contaminantes, como ovos de helmintos, cistos de protozoários e coliformes de origem fecal, e o esterco animal, utilizado como adubo orgânico. Entre as principais espécies de bactérias contaminantes de hortaliças está a Escherichia coli, por apresentar linhagens patogênicas produtoras de toxinas, tais como: hemolisinas, enterotoxinas termolábeis (LT) e termoestáveis (ST) e Shiga-toxina e estar frequentemente associada a surtos de toxinfecções alimentares. E. coli enteropatogênicas (EPEC), apesar de não produzirem Shiga ou verotoxinas, estão entre as linhagens de $\boldsymbol{E}$. coli diarreiogênicas (KAPER et al., 2004).

Considerando-se a relevância e atualidade do problema no Brasil e a crescente participação das hortaliças na transmissão de $\boldsymbol{E}$. coli enteropatogênicas, esta pesquisa teve por objetivo avaliar a atividade antibacteriana do óleo essencial do manjericão
(Ocimum basilicum L.) frente a sorogrupos de $\boldsymbol{E}$. coli enteropatogênica (EPEC) isolados de alfaces (Lactuca sativa) orgânicas e hidropônicas comercializadas em feiras livres e supermercados da cidade de São Luís, Maranhão (MA).

\section{MATERIAL E MÉTODOS}

\section{Coletas das amostras}

Foram coletadas 100 amostras de alfaces (variedade crespa) cultivadas em sistemas orgânico e hidropônico e comercializadas em feiras livres e supermercados, respectivamente, localizados na cidade de São Luís/MA, no período de maio a julho de 2009. A escolha dessa hortaliça se justifica pelo fato de estar entre as mais consumidas pela população maranhense. A opção pelos sistemas de cultivo baseou-se no fato de fornecerem alimentos mais saudáveis, ou seja, livres de agrotóxicos e/ou pesticidas. Após coletadas, as amostras foram acondicionadas em caixas isotérmicas e transportadas ao Laboratório de Microbiologia de Alimentos e Água da Universidade Federal do Maranhão para a realização das análises pertinentes.

Isolamento e identificação das cepas de Escherichia coli

Para o isolamento e a identificação da $\boldsymbol{E}$. coli, utilizou-se a técnica descrita por KORNACKI \& JOHNSON (2001). Para tanto, foram pesados assepticamente $25 \mathrm{~g}$ de cada amostra, que foram incubados em $225 \mathrm{~mL}$ de Caldo Infusão Cérebro Coração (Caldo $\mathrm{BHI}$ ) a $37^{\circ} \mathrm{C}$, por três horas. Após o período de incubação, foram acrescentados ao inóculo 250mL de Caldo Escherichia coli (Caldo E.C.), com posterior incubação a $37^{\circ} \mathrm{C}$, por 24 horas. O plaqueamento seletivo foi realizado nos Agares Eosina Azul de Metileno (EMB) e MacConkey Sorbitol. Após a incubação, as colônias típicas de $\boldsymbol{E}$. coli foram submetidas à identificação bioquímica utilizando-se o Sistema API-20E (bioMérieux).

Identificação sorológica das cepas de Escherichia coli Para a identificação sorológica de $\boldsymbol{E}$. coli enteropatogênicas (EPEC), foram utilizados soros polivalentes e monovalentes (Probac do Brasil ${ }^{\circledR}$ ) contendo anticorpos para a identificação de antígenos somáticos $\mathrm{O}$, a saber: soro polivalente A contendo anticorpos, para a identificação dos sorogrupos monovalentes: O26, O55, O111 e O119; polivalente B, para identificar os monovalentes: 114, O125, O142 e O158; e polivalente C, para os monovalentes: O86, O126, O127 e O128, segundo metodologia descrita por MENG et al. (2001). Para a realização dos testes sorológicos, 
$1 \mathrm{~mL}$ de água estéril foi adicionado às culturas de 24 horas para a obtenção de uma suspensão. Em seguida, foram retiradas alíquotas de $0,1 \mathrm{~mL}$ das respectivas suspensões e adicionadas à superfície de uma lâmina de vidro limpa e seca. Primeiramente, as cepas de $\boldsymbol{E}$. coli foram testadas para os soros polivalentes A, B e C para $\boldsymbol{E}$. coli EPEC clássica e em seguida para os soros monovalentes, de acordo com a classificação obtida nos soros polivalentes. A prova sorológica positiva consistiu da aglutinação do soro após a sua adição na suspensão da cultura bacteriana em estudo.

\section{Extração do óleo essencial}

Para a extração do óleo essencial do manjericão, foram utilizadas as folhas da planta. A extração foi realizada pelo processo de hidrodestilação usando-se o extrator de Clevenger, segundo metodologia descrita por MATOS (1997).

\section{Análise cromatográfica}

Para a identificação dos constituintes do óleo essencial do manjericão, utilizou-se a técnica de Cromatografia a Gás acoplada à Espectrometria de Massas (CG/MS) nas seguintes condições: Equipamento: CGIEM, modelo QP - 5000, fabricado pela Shimadzu; Método: OE_1.met; Amostras: inicialmente pesou-se $1,0 \mathrm{mg}$ de cada amostra e dissolveu-se em $1000 \mu \mathrm{L}$ de diclorometano (pureza 99,9\%); Volume injetado: 0,3 $\mu \mathrm{L}$; Coluna: Capilar, 30mx0,25mmx0,25 $\mu \mathrm{m}$. HP-5MS, 5\% difenil, 95\% dimetil polisiloxano (Equivalente DB-\%MS ou CP-Sil 8CB $\mathrm{LB} \backslash \mathrm{MS})$; Gás de arraste: He (99,9995\%); fluxo: 1,0mL min ${ }^{-1}$; Injetor: $280^{\circ} \mathrm{C}$, modo Split (1:10); Forno: $40^{\circ} \mathrm{C}$ $(5,0 \mathrm{~min}) \rightarrow 240^{\circ} \mathrm{C}\left(4^{\circ} \mathrm{C} \mathrm{min}^{-1}\right) ; 240^{\circ} \mathrm{C} \rightarrow 300^{\circ} \mathrm{C}\left(8^{\circ} \mathrm{C} \mathrm{min}^{-1}\right.$, 7,5min); $\mathrm{t}_{\mathrm{T}}=60,0 \mathrm{~min}$; Detector: EM; EI (70 eV); Espectrômetro de massas tipo quadrupolo linear. Modo de varredura $\left(0,5 \mathrm{~s} \mathrm{sacn}{ }^{-1}\right)$; Faixa de massas: 40-500 daltons; Linha transferência: $280^{\circ} \mathrm{C}$; e Filamento: desligado 0,0 a 4,0min. Para a identificação dos compostos detectados nas amostras, utilizaram-se as bases de dados de espectros de massas NIST105, NIST21 e WILEY139.

\section{Antibiograma}

Para a avaliação da atividade antibacteriana do óleo essencial do manjericão, utilizou-se o Método de Difusão em Disco (MDD), segundo metodologia recomendada pelo NCCLS (2000). Para esse teste, as amostras de $\boldsymbol{E}$. coli EPEC, isoladas das alfaces, foram cultivados em Caldo BHI ( $37^{\circ} \mathrm{C} / 24$ horas) e, após o período de incubação, foram realizadas diluições sucessivas para a obtenção da turbidez de 0,5 na escala de MacFarland $\left(10^{8} \mathrm{UFC} \mathrm{mL}^{-1}\right)$. De cada cultura, retirou- se uma alíquota de $0,1 \mathrm{~mL}$ e inoculou-se na superfície do Agar Müeller-Hinton, sendo utilizado um suabe para espalhar o inóculo. Em seguida, os discos (6mm) impregnados com $75 \mu \mathrm{L}$ do óleo essencial foram colocados no centro da placa com o auxílio de uma pinça. As placas foram incubadas a $37^{\circ} \mathrm{C}$, por 24 horas. A leitura dos halos de inibição foi feita com o auxílio de uma régua milimetrada.

Como parâmetro para a atividade antibacteriana do óleo essencial do manjericão, foram utilizados os padrões de sensibilidade adotados por MOREIRA et al. (2005), os quais classificaram a atividade de diferentes óleos essenciais de acordo com o tamanho do halo de inibição, a saber: não sensível: (-) para diâmetros $\leq 8 \mathrm{~mm}$; sensível: $(+)$ para diâmetros de 9-14mm; muito sensível: $(++)$ para 15-19mm; extremamente sensível: $(+++)$ para os halos com diâmetros $\geq 20 \mathrm{~mm}$.

\section{Análise estatística}

Para a avaliação do efeito inibitório do óleo essencial do manjericão frente aos sorogrupos de $\boldsymbol{E}$. coli EPEC, os dados experimentais foram submetidos ao teste paramétrico para a diferença entre duas médias populacionais (amostras pequenas e independentes) e com um nível de significância de 5\%, sendo utilizado o software STATISTICA 7.0. Esse teste foi realizado supondo-se que as variâncias populacionais $\sigma_{1}^{2}$ e $\sigma_{2}^{2}$ são desconhecidas e estimadas por meio de pequenas amostras $(\mathrm{n} \leq 30)$; $\operatorname{com} \mathrm{n}_{1}+\mathrm{n}_{2}-2$ graus de liberdade. Nesse caso, o critério de decisão para testar a hipótese nula $\mu_{1}-\mu_{2}=d_{0}$ foi baseado no teste $t$-Student, considerandose que as populações são aproximadamente normais.

\section{RESULTADOS E DISCUSSÃO}

Os dados referentes à frequência e ao percentual de isolamento de $\boldsymbol{E}$. coli enteropatogênicas (EPEC) das amostras de alfaces cultivadas pelos sistemas orgânico e hidropônico estão expressos na tabela 1. De acordo com os resultados, independentemente do sistema de cultivo (orgânico ou hidropônico), foram identificados, por meio dos testes sorológicos para $\boldsymbol{E}$. coli enteropatogênica clássica EPEC, em ambas as amostras de alface, os sorogrupos: O55, O111, O114, O125, O127 e O142.

No Brasil, estima-se que as diarréias sejam as responsáveis por mais de 200.000 óbitos anuais de crianças, nos quais a EPEC se encontra entre as principais causas. A infecção por EPEC é transmitida principalmente pelo consumo de água ou alimentos contaminados, sendo o problema agravado quando há imunodreprimidos (TRABULSI et al., 2002). 
Tabela 1 - Frequência e percentual de isolamento de Escherichia coli enteropatogênicas isoladas das amostras de alfaces cultivadas pelos sistemas de cultivo orgânico e hidropônico comercializadas em feiras livres e supermercados da cidade de São Luís/ MA.

\begin{tabular}{|c|c|c|c|c|c|c|c|}
\hline \multirow{3}{*}{ Amostra } & \multirow{3}{*}{ Cultivo } & \multicolumn{6}{|c|}{ 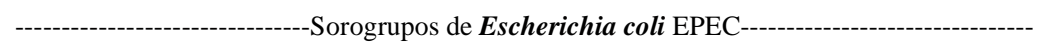 } \\
\hline & & O55 & O111 & O114 & O125 & O127 & O142 \\
\hline & & $\mathrm{n}(\%)$ & $\mathrm{n}(\%)$ & $\mathrm{n}(\%)$ & $\mathrm{n}(\%)$ & $\mathrm{n}(\%)$ & $\mathrm{n}(\%)$ \\
\hline \multirow{4}{*}{ Alface } & \multirow{2}{*}{ Orgânico } & 29 & 1 & 2 & 9 & 1 & 7 \\
\hline & & $(59,2)$ & $(2,0)$ & $(4,1)$ & $(18,3)$ & $(2,0)$ & $(14,3)$ \\
\hline & \multirow{2}{*}{ Hidropônico } & 6 & 3 & 2 & 2 & 1 & 2 \\
\hline & & $(37,5)$ & $(18,8)$ & $(12,5)$ & $(12,5)$ & $(6,2)$ & $(12,5)$ \\
\hline
\end{tabular}

Nota: n: Número de $\boldsymbol{E}$. coli positivas para os sorogrupos de EPEC clássica; (\%): percentual de isolamento.

Diversos surtos de toxinfecções alimentares têm sido associados com hortaliças contaminadas. Pesquisadores têm recuperado várias bactérias patogênicas para humanos desses produtos, tais como Listeria monocytogenes, Salmonella sp. e E. coli O157:H7 cultivadas em ambos os sistemas de produção - orgânico e hidropônico. Entre as principais fontes de contaminação das hortaliças por linhagens de $\boldsymbol{E}$. coli enteropatogênicas estão o esterco, utilizado com adubo orgânico, e a água de irrigação contaminada, utilizada no sistema de produção orgânico, bem como a manipulação, os nutrientes e a água contendo material fecal na produção hidropônica (McMAHON \& WILSON, 2001). SAFARIKOVÁ \& SAFARIK (2001), ao avaliarem a contaminação de amostras de hortaliças, incluindo a alface, constataram a contaminação destas por sorogrupos de $\boldsymbol{E}$. coli O26, O111 e O157.

A análise por CG/EM do óleo essencial do manjericão estudado nesta pesquisa identificou seis componentes como sendo os principais constituintes do óleo com um percentual de abundância significativo, a saber: o metil chavicol ou estragol [1-metoxi-4-(2- propenil)-benzeno] (62,39\%), linalol (25,88\%), $\alpha$ farneseno (6,14\%), 1,8 cineol (eucaliptol) (3,48\%), $\alpha$ terpineol [4-metil-1-(1-metiletil)-3-ciclohexen-1-ol] (1,43\%) e $\alpha$-cadinol $(0,67 \%)$.

Nesta pesquisa, sorogrupos de $\boldsymbol{E}$. coli EPEC isolados das amostras de alfaces foram testados frente à ação antibacteriana do óleo essencial do manjericão, em que as características numéricas obtidas a partir do antibiograma realizado com o óleo essencial estão apresentadas nas tabelas 2 e 3. De acordo com os resultados, os sorogrupos de $\boldsymbol{E}$. coli EPEC testados demonstraram sensibilidade $(+)$ frente à ação do óleo essencial do manjericão, com médias de halos de inibição variando entre $11,2 \mathrm{~mm}$ e $11,6 \mathrm{~mm}$ e de $11,0 \mathrm{~mm}$ a 12,0mm para os isolados das alfaces orgânicas e hidropônicas, respectivamente. Após a análise estatística aplicada a esses resultados com a finalidade de comparar as médias dos halos de inibição, não foram constatadas diferenças significativas $(\mathrm{P}>0,05)$ entre as cepas de $\boldsymbol{E}$. coli EPEC sensíveis ao óleo de manjericão. O efeito inibitório apresentado pelo óleo do manjericão observado nesta pesquisa foi atribuído à

Tabela 2 - Características numéricas da atividade antibacteriana do óleo essencial do manjericão frente às cepas de Escherichia coli EPEC clássica isoladas de alfaces cultivadas pelo sistema de cultivo orgânico, segundo sorogrupos e tamanho do halo de inibição (mm).

\begin{tabular}{|c|c|c|c|c|c|}
\hline Sorogrupos & Tamanho da amostra & $\begin{array}{l}\text { Média aritmética } \\
\text { (Halo/mm) }\end{array}$ & $\begin{array}{c}\text { Intervalo de } \\
\text { confiança 95\% }\end{array}$ & Desvio padrão & $\begin{array}{c}\text { Coeficiente de } \\
\text { variação (\%) }\end{array}$ \\
\hline 055 & 29 & $11,2 \mathrm{a}$ & 11,08 a 11,41 & 0,4355 & 3,87 \\
\hline 0111 & 5 & $11,4 \mathrm{a}$ & 10,72 a 11,09 & 0,5477 & 4,80 \\
\hline 0114 & 10 & 11,6 a & 11,23 a 11,97 & 0,5163 & 4,45 \\
\hline 0125 & 9 & $11,2 \mathrm{a}$ & 10,88 a 11,56 & 0,4410 & 3,93 \\
\hline 0127 & 5 & $11,4 \mathrm{a}$ & 10,72 a 12,08 & 0,5477 & 4,80 \\
\hline 0142 & 7 & $11,4 \mathrm{a}$ & 10,93 a 11,92 & 0,5345 & 4,68 \\
\hline
\end{tabular}

Nota: Médias seguidas com mesma letra minúscula, na mesma linha, não diferem significativamente entre si a 95\% de probabilidade pelo teste t de Student $(\mathrm{P}<0,05)$; (-) Não há variação entre as variáveis.

Ciência Rural, v.40, n.8, ago, 2010. 
Tabela 3 - Características numéricas da atividade antibacteriana do óleo essencial do manjericão frente às cepas de Escherichia coli EPEC clássica isoladas de alfaces cultivadas pelo sistema de cultivo hidropônico, segundo sorogrupos e tamanho do halo de inibição (mm).

\begin{tabular}{|c|c|c|c|c|c|}
\hline Sorogrupos & Tamanho da amostra & $\begin{array}{l}\text { Média aritmética } \\
\text { (Halo/mm) }\end{array}$ & $\begin{array}{l}\text { Intervalo de confiança } \\
\qquad 95 \%\end{array}$ & Desvio padrão & $\begin{array}{c}\text { Coeficiente de variação } \\
\text { (\%) }\end{array}$ \\
\hline 055 & 30 & $11,7 \mathrm{a}$ & 11,49 a 11,85 & 0,4795 & 4,11 \\
\hline 0111 & 15 & $11,0 \mathrm{a}$ & - & 0,00 & 0,00 \\
\hline 0114 & 10 & $11,5 \mathrm{a}$ & 11,12 a 11,88 & 0,5271 & 4,58 \\
\hline 0125 & 10 & 11,9 a & 11,67 a 12,13 & 0,3162 & 2,66 \\
\hline 0127 & 5 & $11,0 \mathrm{a}$ & - & 0,00 & 0,00 \\
\hline 0142 & 10 & 12,0 a & - & 0,00 & 0,00 \\
\hline
\end{tabular}

presença do metil chavicol e linalol, os constituintes majoritários do óleo. Esse resultado é semelhante aos achados de WAN et al. (1998), quando avaliaram a ação antibacteriana do óleo essencial dessa espécie frente a uma variedade de bactérias gram positivas e gram negativas, incluindo a $\boldsymbol{E}$. coli. Os resultados aqui apresentados concordam ainda com os achados por DUARTE et al. (2007), os quais, estudando o efeito de óleos essenciais de plantas medicinais usadas no Brasil, incluindo o manjericão, frente a $\boldsymbol{E}$. coli enteropatogênicas (EPEC) e $\boldsymbol{E}$. coli enterotoxigênicas (ETEC), classificaram a atividade antibacteriana do óleo essencial do manjericão como sendo de atividade moderada.

HUSSAIN et al. (2008) relataram a ação do óleo essencial de manjericão frente à Escherichia coli, a qual demonstrou sensibilidade ao apresentar um halo de inibição de 11,4mm. BOZIN et al. (2006), caracterizando a composição dos constituintes voláteis dos óleos essenciais de algumas espécies da família Lamiaceae, bem como a sua atividade antimicrobiana, verificaram um halo de inibição de $11 \mathrm{~mm}$ de diâmetro para o manjericão frente a $\boldsymbol{E}$. coli multirresistentes a antibióticos comerciais. Os autores atribuíram o efeito inibitório à presença dos constituintes majoritários do óleo, o metil chavicol e o linalol.

SOKOVIC \& VAN GRIENSVEN (2006) comentaram que o manjericão tipo metil chavicol apresentou significante atividade contra o crescimento de diferentes microrganismos, incluindo a $\boldsymbol{E}$. coli, resultados que corroboram os achados nesta pesquisa. Os referidos autores relataram ainda que a atividade antimicrobiana do óleo essencial de manjericão pode ser atribuída ao linalol e a outros compostos oxigenados.

\section{CONCLUSÃO}

O teste in vitro do óleo essencial do manjericão testado frente a diferentes sorogrupos de
E. coli EPEC clássica mostrou atividade antibacteriana significante, sugerindo que este pode ser utilizado como uma alternativa no combate as toxinfecções causadas por $\boldsymbol{E}$. coli. Porém, o potencial uso desse óleo como fitoterápico no tratamento de doenças infecciosas necessita de avaliação in vivo e de novas pesquisas excluindo possíveis efeitos colaterais para a fisiologia digestiva e microbiota normal de indivíduos infectados.

\section{REFERÊNCIAS}

BOZIN, B. et al. Characterization of the volatile composition of essential oils of some Lamiaceae species and the antimicrobial and antioxidant activities of the entire oils. Journal of Agricultural Food Chemistry, v.54, n.5, p.1822-1828, 2006. Disponível em: <http://pubs.acs.org/doi/pdf/10.1021/jf051922u>. Acesso em: 20 fev. 2009. doi: 10.1021/jf051922u.

DUARTE, M.C.T. et al. Activity of essential oils from Brazilian medicinal plants on Escherichia coli. Journal of Ethnopharmacology. v. 111, n. 2, p. 197-201, 2007. Disponível em: <http://www.sciencedirect.com/ science?_ob=ArticleURL\&_udi=B6T8D-4MGM2RR1 \&_user $=686379 \&$ _coverDate $=05 \% 2$ F04\%2F2007\&_rdoc $=1$ \&_fmt=high\&_orig=search\&_sort $=$ d\&_docanchor $=$ \& vie $\mathrm{w}=\mathrm{c} \&$ _ a c c t $=$ C $000037538 \&$ \&_version $=1 \&$ \&_url V ersion=0\&_userid=686379\&md5=7716c11416d2e95499108d3e7b84ffd4>. Acesso em: 15 mar. 2009. doi: 10.1016/j.jep.2006.11.034.

FERNANDES, A.A. et al. Produtividade, acúmulo de nitrato e estado nutricional de cultivares de alface, em hidropônia, em função de fontes de nutrientes. Horticultura Brasileira, v.20, n.2, p.195-200, 2002. Disponível em: <http:// w w w. s c i e l o.b r / s c i e lo.p h p ? p i d = S 0102 05362002000200016\&script=sci_arttext $>$. Acesso em: 26 mar. 2009. doi: 10.1590/S0102-05362002000200016.

HUSSAIN, A.I. et al. Chemical composition, antioxidant and antimicrobial activities of basil (Ocimum basilicum L.) essential oils depends on seasonal variations. Food Chemistry, v. 108, n.3, p. 986-995, 2008. Disponível em: <http:// $\mathrm{w}$ w w. S c i e $\mathrm{n}$ c e d i r e c t c c c m / science?_ob=ArticleURL\&_udi=B6T6R-4RB Y G2H$8 \&$ _user $=686379 \&$ \&coverDate $=06 \% 2 \mathrm{~F} 01 \% 2 \mathrm{~F} 2008 \&$ \& rdoc $=1 \&$ _fmt $=$ high\&_orig $=$ search \&_sort $=$ d $\& \_$docanchor $=\& v i e w=c$ 
\&_acct $=$ C000037538\&_version $=1 \&$ \&_urlVersion $=0 \&$ \&u serid=686379\&md5=bd7c7f42a97e9044cfc26d7d03227a39>. Acesso em: 02 abr. 2009. doi: 10.1016/j.foodchem.2007.12.010.

KAPER, J.B. et al. Pathogenic Escherichia coli. Nature Reviews Microbiology, v.2, p.123-140, 2004. Disponível em: <http://www.nature.com/nrmicro/journal/v2/n2/pdf/ nrmicro818.pdf>. Acesso em: 02 abr. 2009. doi: 10.1038/ nrmicro818.

KORNACKI, J.L.; JOHNSON, J.L. Enterobateriaceae, coliforms and Escherichia coli as quality and safety indicators. In: VANDERZANT, C.; SPLITTSTOESSER, D.F. Compendium of methods for the microbiological examination of foods. 4.ed. Washington: APHA - American Public Health Association, 2001. Cap.8, p.69-82.

LABRA, M. et al. Morphological characterization, essential oil composition and DNA genotyping of Ocimum basilicum L. cultivars. Plant Science, v.167, p.725-731, 2004. Disponível em: <http:// www.sciencedirect.com/science?_ob=ArticleURL\&_udi=B6TBH4CG0MC1- 1\&_user=686379\&_coverDate $=10 \% 2 F 31 \% 2 F 2004 \&$ rd oc $=1 \& \_$fmt $=$high \&_orig $=$search \&_sort $=$d\&_docanchor $=\&$ view $=$ c\&_acct $=$ C000037538\&_version $=1 \&$ \&urlVersion $=0$ \&_userid $=686379 \& \mathrm{md} 5=\mathrm{c} 92 \mathrm{ce} 5764 \mathrm{~d} 11 \mathrm{de} 096959 \mathrm{e} 809 \mathrm{f} 8211357>$. Acesso em: 20 abr. 2009. doi: 10.1016/j.plantsci.2004.04.026.

McMAHON, M.A.S.; WILSON, I.G. The occurrence of enteric pathogens and Aeromonas species in organic vegetables. International Journal of Food Microbiology, v.70, n.1-2, p.155162, 2001. Disponível em: <http://www.sciencedirect.com/ science?_ob=ArticleURL\&_udi=B6T7K-44724 S4J\&_user $=686379 \&$ _coverDate $=10 \% 2$ F 22\%2F 2001\&_ rdoc $=1 \& \_$fmt $=$high\&_orig $=$search\&_sort $=\mathrm{d} \&$ _docanchor $=\&$ view $=\mathrm{c} \&$ _acct $=$ C $000037538 \&$ \&version $=1 \&$ \&urlVersion $=0 \&$ \&us erid $=686379 \& \mathrm{md} 5=1 \mathrm{f} 522 \mathrm{a} 487 \mathrm{e} 0 \mathrm{fb} 928 \mathrm{e} 43 \mathrm{a} 3 \mathrm{~d} 0887 \mathrm{c} 2 \mathrm{ff} 8 \mathrm{a}>$. Acesso em: 12 abr. 2009. doi: 10.1016/S0168-1605(01)00535-9.

MATOS, F.J.A. Introdução à fotoquímica experimental. 2.ed. Fortaleza: UFC, 1997. 141p.

MENG, J. et al. Pathogenic Escherichia coli. In: VANDERZANT, C.; SPLITTSTOESSER, D.F. Compendium of methods for the microbiological examination of foods. 4.ed. Washington: APHA - American Public Health Association, 2001. Cap.35, p.331-341.

MOREIRA, M.R. et al. Inhibitory parameters of essential oils to reduce a foodborne pathogen. LWT- Food Science and Techonology, v.38, n.5, p.565-570, 2005. Disponível em: $<\mathrm{ht} \mathrm{t}$ : :// w w w. s c i e n c ed i r e c t. c o m/ science?_ob=ArticleURL\&_udi=B6WMV-4FR3GG6$2 \&$ \&user $=686379 \&$ \&coverDate $=08 \% 2 \mathrm{~F} 31 \% 2 \mathrm{~F} 2005 \&$ \&doc $=1$ \&_fmt=high\&_orig=search\&_sort $=$ d\&_docanchor $=\&$ view $=c \&$ \&_act $=$ C $000037538 \&$ \&_version $=1 \&$ \&_urlVersion $=0 \&$ _userid=686379\&md5=dbde48e0cd7504ea282404df8fb45743>. Acesso em: 12 abr. 2009. doi: 10.1016/j.lwt.2004.07.012.
NATIONAL COMMITTEE FOR CLINICAL LABORATORY STANDARDS - NCCLS. Performance standards for antimicrobial disk susceptibility tests. 7.ed. Wayne, $\mathrm{Pa}$ : National Committee for Clinical Laboratory Standards, 2000. (Approved Standard M2-A7).

SAFARIKOVÁ, M.; SAFARIK, I. Immunomagnetic separation of Escherichia coli O26, O111 and O157 from vegetables. Letters in Applied Microbiology, v.33, p.36-39, 2001. Disponível em: <http://www3.interscience.wiley.com/cgi-bin/ fulltext/118975535/PDFSTART>. Acesso em: 15 abr. 2009. doi: 10.1046/j.1472-765X.2001.00941.x.

SANTANA, L.R.R. et al. Qualidade física, microbiológica e parasitológica de alfaces (Lactuca sativa) de diferentes sistemas de cultivo. Ciência e Tecnologia de Alimentos, v.26, n.2, p.264-269, 2006. Disponível em: <http://www.scielo.br/pdf/ cta/v26n2/30171.pdf $>$. Acesso em: 15 mar. 2009. doi: 10.1590/S0101-20612006000200006.

SARTORATTO, A. et al. Composition and antimicrobial activity of essential oils from aromatic plants used in Brazil. Brazilian Journal Microbiology, v.35, n.4, p.273-280, 2004. Disponível em: <http://www.scielo.br/pdf/bjm/v35n4/ v35n4a01.pdf>. Acesso em: 10 mar. 2009. doi: 10.1590/ S1517-83822004000300001.

SOKOVIC, M.; VAN GRIENSVEN, L.J.L.D. Antimicrobial activity of essential oils and their components against the three major pathogens of the cultivated button mushroom, Agaricus bisporus. European Journal Plant Pathology, v.116, n.3, p.211-224, 2006. Disponível em: <http://www.springerlink.com/ content/t285j32876337950/fulltext.pdf $>$. Acesso em: 10 mar. 2009. doi: 10.1007/s10658-006-9053-0.

TELCI, I. et al. Variability in essential oil composition of Turkish basils (Ocimum basilicum L.). Biochemical Systematics and Ecology, v.34, n.6, p.489-497, 2006. Disponível em: <http:// www.sciencedirect.com/science?_ob=ArticleURL\&_udi=B6T4R4JHMFR6- $1 \&$ _user $=686379 \&$ \&_coverDate $=06 \% 2$ F30\%2F20 $06 \&$ \& rdoc $=1 \&$ \&_fmt $=$ high\&_orig=search\&_sort $=$ d \& docanchor $=\&$ vie w $=$ c \&_a c ct $=$ C $000037538 \&$ \& ersion $=1 \&$ \&_url Version $=0 \&$ \&_userid $=686379 \&$ md5=33791afda2c0dc378dcf2d6a951e9790>. Acesso em: 11 abr. 2009. doi: 10.1016/j.bse.2006.01.009.

TRABULSI, L.R. et al. Typical and atypical enteropathogenic Escherichia coli. Emerging Infectious Diseases Journal, v.8, n.5, p.508-513, 2002. Disponível em: <http://www.cdc.gov/ Ncidod/eid/vol8no5/pdf/01-0385.pdf>. Acesso em: $11 \mathrm{abr}$. 2009. doi: 10.3201/eid0805.010385.

WAN, J. et al. The effect of essential oils of basil on the growth of Aeromonas hydrophila and Pseudomonas fluorescens. Journal of Applied Microbiology, v.84, n.2, p.152-158, 1998. Disponível em: <http://www3.interscience.wiley.com/cgi-bin/ fulltext/119136352/PDFSTART>. Acesso em: 11 abr. 2009. doi: 10.1046/j.1365-2672.1998.00338.x. 